\title{
AN EXTREMAL PROBLEM IN GRAPH THEORY II
}

\author{
H. L. ABBOTT, M. KATCHALSKI and A. C. LIU
}

(Received 25 June; revised 14 November 1979)

Communicated by W. D. Wallis

\begin{abstract}
We continue our study of the following combinatorial problem: What is the largest integer $N=N(t, m, p)$ for which there exists a set of $N$ people satisfying the following conditions: (a) each person speaks $t$ languages, (b) among any $m$ people there are two who speak a common language and (c) at most $p$ speak a common language. We obtain bounds for $N(t, m, p)$ and evaluate $N(3, m, p)$ for all $m$ and infinitely many values of $p$.
\end{abstract}

1980 Mathematics subject classification (Amer. Math. Soc.) : 05 C 35.

\section{Introduction}

We continue in this paper the study of the following combinatorial problem, begun in Abbott and others (1980). Let $t, m \geqslant 2$ and $p \geqslant 2$ be positive integers. What is the largest integer $N=N(t, m, p)$ for which there exists a set of $N$ people satisfying the following conditions : (a) each person speaks $t$ languages, (b) among any $m$ people, two speak a common language and (c) at most $p$ speak a common language?

In Abbott and others (1980) it was shown that

$$
\begin{aligned}
& N(t, m, p) \leqslant(m-1)(t p-t+1), \\
& N(t, m, p) \geqslant \begin{cases}\frac{1}{2} p(m-1)(t+1) & \text { if } p \text { is even, } \\
\frac{1}{2}(m-1)(t p-t+p+1) & \text { if } p \text { is odd, }\end{cases}
\end{aligned}
$$

and

$$
N(2, m, p)= \begin{cases}\frac{3}{2}(m-1) p & \text { if } p \text { is even, } \\ \frac{1}{2}(m-1)(3 p-1) & \text { if } p \text { is odd. }\end{cases}
$$

Note that when $t=1$ or when $p=2$, the upper and lower bounds given by (1) and (2) coincide. It was further shown that equality holds in (1) whenever there exists a block design $B(b, v, r, k, \lambda)$ with $r=t, k=p, \lambda=1$. 
For large values of the arguments, the bounds given by (1) and (2) differ roughly by a factor of 2 . In this paper we prove the following result which shows that for $t$ large and $p$ large compared to $t$, the bound given by (1) is asymptotically correct.

THEOREM 1. For each $\varepsilon>0$ there exists a number $t_{0}=t_{0}(\varepsilon)$ such that for $t \geqslant t_{0}$ and $p>2 t / \varepsilon$,

$$
N(t, m, p)>(1-\varepsilon) t p(m-1) .
$$

We also determine $N(3, m, p)$ for all $m \geqslant 2$ and infinitely many values of $p$ by showing that the following result holds :

THEOREM 2. For all $m \geqslant 2$ and all $p \geqslant 2$,

$$
N(3, m, p) \leqslant \frac{7}{3}(m-1) p
$$

Furthermore, equality holds in $(5)$ when $p \equiv 0(\bmod 3)$.

Finally, we consider a variation of the problem which was suggested to us by A. Meir.

\section{Proof of Theorem 1}

We shall need the following lemma.

LEMMA 1. Let $t$ be such that a finite projective plane of order $t-1$ exists and suppose that $p=t k$ for some integer $k$. Then

$$
N(t, m, p) \geqslant\left(t^{2}-t+1\right)(m-1)(p / t) .
$$

Proof. Take $m-1$ disjoint copies of a finite projective plane of order $t-1$. Let each line denote a distinct language and each point a set of $k$ people all of whom speak the $t$ languages represented by lines incident with that point. There are then $\left(t^{2}-t+1\right)(m-1)(p / t)$ people and it is easy to check that this group satisfies (a), (b) and (c). This completes the proof of the lemma.

It is now a simple matter to deduce Theorem 1. Projective planes of order $t-1$ are known to exist only when $t-1$ is a prime power. Let $q-1$ be the largest prime power not exceeding $t-1$ and let $s q$ be the largest multiple of $q$ not exceeding $p$. Then by Lemma 1 and the fact that $N$ is nondecreasing in $t$ and $p$ we get

$$
N(t, m, p) \geqslant N(q, m, s) \geqslant\left(q^{2}-q+1\right)(m-1)(s / q) \text {. }
$$


By the Prime Number Theorem, if $\varepsilon>0$ is given, there exists a prime power $q-1$ satisfying $\left(1-\frac{1}{2} \varepsilon\right) t<q-1<t$ for all sufficiently large $t$. It follows from this, the condition $p>2 t / \varepsilon$ and (7) that (4) holds. We suppress the details of the routine calculation involved. This completes the proof of Theorem 1.

We remark that D. Hanson has informed us that he and J. C. Fisher obtained Lemma 1 also.

\section{Proof of Theorem 2}

It will be convenient to formulate the problem in more abstract terms. It is clear that $N=N(t, m, p)$ is the largest integer for which there exists a family $\mathscr{F}$ of $N$ sets, not necessarily distinct, satisfying the following conditions : (a)' each member of $\mathscr{F}$ has $t$ elements, (b)' among any $m$ members of $\mathscr{F}$ there are two with nonempty intersection and (c) $)^{\prime}$ at most $p$ members of $\mathscr{F}$ have nonempty intersection. The elements of $\bigcup \mathscr{F}$ correspond to the languages and the members of $\mathscr{F}$ to people; so that if $a \in F$, the person corresponding to $F$ speaks language $a$. That the members of $F$ need not be distinct simply reflects the fact that two or more people may speak the same set of languages. Note that according to (c)', however, a member of $\mathscr{F}$ appears at most $p$ times.

It will also be necessary in what follows to allow a set to have elements appearing more than once. Such a set will be called a multiset and the number of times an element appears will be called its multiplicity. We remark that the cardinality of a multiset (or family) takes into account the multiplicity of the elements (or members).

We need the following simple, but important, observation which we record as a lemma.

LEMMA 2. Let $\mathscr{F}$ be a family of sets satisfying (c)'. Suppose there is a multiset $L$ such that $|L \cap F| \geqslant t$ for each $F \in \mathscr{F}$ (we say that $L$ covers $\mathscr{F} t$ times). Then

$$
|\mathscr{F}| \leqslant \frac{|L| p}{t} .
$$

Now we prove Theorem 2 . Let $\mathscr{F}$ be a maximal family satisfying (a)', (b)' and (c)' with $t=3$, so that $|\mathscr{F}|=N(3, m, p)$. We may suppose there exists a subfamily $\mathscr{T}=\left\{F_{1}, F_{2}, \ldots, F_{m-1}\right\}$ of pairwise disjoint members of $\mathscr{F}$, since otherwise $\mathscr{F}$ would not be maximal. Let $F_{i}=\left\{a_{i}, b_{i}, c_{i}\right\}$ and let $A=\bigcup F_{i}$.

For $i=1,2, \ldots, m-1$, let $B_{i}$ be a multiset of at most four elements, to be specified later. Let $C_{1}=\emptyset$ and let $C_{i}=C_{i-1} \oplus B_{i}$ for $2 \leqslant i \leqslant m-1$, where $\oplus$ denotes union with multiplicities. Then $C_{m}$ is a multiset of size at most $4(m-1)$ and $A \oplus C_{m}$ is a multiset of size at most $7(m-1)$. We shall show that it is possible to define the 
multisets $B_{i}$ in such a way that $A \oplus C_{m}$ covers $\mathscr{F}$ three times. The theorem will then follow from Lemma 2.

For each $i, 1 \leqslant i \leqslant m-1$, let

$$
\begin{aligned}
\mathscr{S}_{i}^{(1)} & =\left\{F: F \in \mathscr{F},\left|F \cap F_{i}\right|=1, F \cap F_{j}=\emptyset \text { for } j \neq i\right\}, \\
\mathscr{S}_{i}^{(2)} & =\left\{F: F \in \mathscr{F},\left|F \cap F_{i}\right|=2, F \cap F_{j}=\emptyset \text { for } j \neq i\right\}
\end{aligned}
$$

and for each pair $i, j, 1 \leqslant i<j \leqslant m-1$, let

$$
\mathscr{S}_{i j}=\left\{F: F \in \mathscr{F},\left|F \cap F_{i}\right|=\left|F \cap F_{j}\right|=1, F \cap F_{k}=\emptyset \text { for } k \neq i, j\right\} .
$$

It suffices, in constructing the multisets $B_{i}$, to ensure that (i) $C_{m}$ covers $\mathscr{S}_{i}^{(1)}$ twice, (ii) $C_{m}$ covers $\mathscr{S}_{i}^{(2)}$ once and (iii) $C_{m}$ covers $\mathscr{S}_{i j}$ once.

Note that for each $i$ any two members of $\mathscr{S}_{i}^{(1)} \cup \mathscr{S}_{i}^{(2)}$ have nonempty intersection, since otherwise we may replace $F_{i}$ in $\mathscr{T}$ by two members of $\mathscr{S}_{i}^{(1)} \cup \mathscr{S}_{i}^{(2)}$ to get $m$ pairwise disjoint members of $\mathscr{F}$, contrary to (b)'. This observation will be used frequently in what follows, often without direct mention.

We now construct $B_{i}$. Let $G_{i}$ be the graph whose vertex set is

$$
V_{i}=\left\{x: x \in E-F_{i}, E \in \mathscr{S}_{i}^{(1)}\right\}
$$

and let two vertices $x, y \in V_{i}$ be joined by an edge if $x, y \in E$ for some $E \in \mathscr{S}_{i}^{(1)}$. $B_{i}$ is defined according to the structure of $G_{i}$, and we will check that conditions (i) and (ii) are satisfied as we choose $B_{i}$. Conditions (i) and (ii) are obviously satisfied if $F_{i} \subseteq B_{i}$. In fact, in such cases $B_{i}$ and hence also $C_{m}$ will cover $\mathscr{S}_{i j}$ once for any $j, i<j \leqslant m-1$. The verification that (iii) is satisfied when $F_{i} \ddagger B_{i}$ will be given after the construction of $C_{m}$ is completed. To simplify the notation we drop the subscript $i$ until further notice and write $a, b, c, F, B, G, \mathscr{S}^{(1)}$ and $\mathscr{S}^{(2)}$.

Case 1. $G$ is empty. Choose $B=\{a, b, c\}$.

Case 2. $G$ is a star around vertex $w$. Choose $B=\{a, b, c, w\}$.

Case 3. $G$ is a triangle with vertices $x, y, z$. If $\{x, y, z\} \cap C \neq \emptyset$, say $z \in C$, choose $B=\{a, b, c, x\}$. We may therefore suppose $\{x, y, z\} \cap C=\emptyset$. For definiteness and without loss of generality, suppose that $\{a, x, y\} \in \mathscr{P}^{(1)}$. Then choose $B=\{a, x, y, z\}$. It is clear that $B$ covers $\mathscr{S}^{(1)}$ twice. If $\mathscr{S}^{(2)}$ were not covered once by $B$, there would exist $\{b, c, u\} \in \mathscr{S}^{(2)}, u \neq x, y, z$. But then $\{a, x, y\} \cap\{b, c, u\}=\varnothing$ contrary to the observation made earlier. Thus $B$ covers $\mathscr{S}^{(2)}$ once. For the purpose of verifying (iii) later, we label $B=\{a, x, y, z\}$ Type 1 .

Case 4. $G$ contains two independent edges, say $(w, x)$ and $(y, z)$. Then, without loss of generality, we may suppose that $\{a, w, x\}$ and $\{a, y, z\}$ are members of $\mathscr{S}^{(1)}$. Let $H$ 
be the subgraph of $G$ in which two vertices $u, v$ are joined by an edge if $u, v \in E$ for some $E \in \mathscr{T}^{(1)}$ not containing $a$. Then the edge set of $H$ is a subset of $\{(w, y),(w, z),(x, y)(x, z)\}$, since otherwise (b)' would be contradicted. Note also that any member $E$ of $\mathscr{S}^{(1)}$ which contains a must contain a vertex of each edge of $H$ since otherwise (b)' would again be violated. We need to distinguish several subcases.

Case $4 a . H$ is empty. Choose $B=\{a, a, b, c\}$.

Case $4 b . H$ consists of a single edge, say $(w, y)$. Suppose, without loss of generality, that $\{b, w, y\} \in \mathscr{S}^{(1)}$. If $\{w, y\} \cap C \neq \varphi$, say $y \in C$, choose $B=\{a, b, c, w\}$. If $\{w, y\} \cap C=\emptyset$ we may choose $B=\{a, b, w, y\}$. For the purpose of verifying (iii) later, label $B=\{a, b, w, y\}$ Type 2 .

Case $4 c . H$ consists of two connected edges, say $(w, y)$ and $(w, z)$. If $\{y, z\} \cap C \neq \emptyset$, we may choose $B=\{a, b, c, w\}$. If $\{y, z\} \cap C=\varnothing$ and $w \in C$ we may choose $B=\{a, b, c, y\}$. If $\{w, y, z\} \cap C=\emptyset$. we choose $B=\{a, w, y, z\}$. It is clear that $B$ covers $\mathscr{S}^{(1)}$ twice. If $\mathscr{S}^{(2)}$ were not covered once there would exist $\{b, c, u\} \in \mathscr{S}^{(2)}$, $u \neq y, z$. Then $\{a, y, z\} \cap\{b, c, u\}=\varphi$, contrary to our earlier observation. Thus $B$ covers $\mathscr{S}^{(2)}$ once. For the purpose of verifying (iii) we label $B=\{a, w, y, z\}$ Type 3 .

Case $4 d$. $H$ contains two independent edges, say $(w, y)$ and $(x, z)$. We may assume, without loss of generality, that $\{w, y, b\}$ and $\{x, z, b\}$ are in $\mathscr{S}^{(1)}$. Note then that if $\mathscr{S}^{(2)}$ is nonempty, every number of $\mathscr{S}^{(2)}$ contains $a$ and $b$. If at least two of $w, x, y, z$ are in $C$, we may choose $B$ such that $F \subseteq B$ : Hence we may suppose that at most one of $w, x, y, z$ is in $C$. We need to consider two sub-cases.

Case $4 d(i) . \mathscr{S}^{(2)}$ does not contain a set $\{a, b, u\}$ with $u \notin\{w, x, y, z\}$. Then we may choose $B=\{w, x, y, z\}$. For the purpose of verifying (iii), label $B=\{w, x, y, z\}$ Type 4 .

Case $4 d(i i) . \mathscr{S}^{(2)}$ contains a set $\{a, b, u\}$ with $u \notin\{w, x, y, z\}$. Then we may choose $B=\{a, a, b, b\}$, which we label Type 5 .

This completes the construction of $B_{i}$ and hence also of $C_{m}$. We now have to verify (iii). It will be necessary to reintroduce the subscripts. Also, of course, certain symbols, especially $w, x, y, z$, introduced in the preceding discussion, will now appear with subscripts. Suppose there exist subscripts $i$ and $j, 1 \leqslant i<j \leqslant m-1$ such that $\mathscr{S}_{i j}$ contains a set $\left\{d_{i}, d_{j}, r\right\}$ where $d_{i} \in F_{i}$ and $d_{j} \in F_{j}$ and $\left\{d_{i}, d_{j}, r\right\} \cap C_{m}=\emptyset$. The idea is to show that we may replace $F_{i}$ and $F_{j}$ in $\mathscr{T}$ by three sets so as to obtain $m$ members of $\mathscr{F}$ which are pairwise disjoint, in contradiction to (b)'. How this may be done is indicated in Table 1 . We single out for detailed explanation only the case 
TABLE 1

Type of Type of

$B_{i} \quad B_{j} \quad$ Replacements for $F_{i}$ and $F_{j}$

\begin{tabular}{|c|c|c|c|}
\hline & 1 & $\left\{d_{i}, d_{j}, r\right\}$ & $\left\{a_{i}, x_{i}, y_{i}\right\} \quad\left\{a_{j}, x_{j}, y_{j}\right\}$ \\
\hline 1 & 2 & $\left\{d_{i}, d_{j}, r\right\}$ & $\left\{a_{i}, x_{i}, y_{i}\right\} \quad\left\{b_{j}, w_{j}, y_{j}\right\}$ \\
\hline 1 & 3 & $\left\{d_{i}, d_{j}, r\right\}$ & $\left\{a_{i}, x_{i}, y_{i}\right\} \quad\left\{a_{j}, y_{j}, z_{j}\right\}$ \\
\hline 1 & 4 & $\begin{array}{l}\left\{d_{i}, d_{j}, r\right\} \\
\left\{d_{i}, d_{j}, r\right\}\end{array}$ & $\begin{array}{r}\left\{a_{i}, x_{i}, y_{i}\right\} \quad\left\{a_{j}, w_{j}, x_{j}\right\} \text { if } d_{j} \neq a_{j} \\
\left\{a_{i}, x_{i}, y_{i}\right\} \quad\left\{b_{j}, w_{j}, y_{j}\right\} \text { if } d_{j}=a_{j}\end{array}$ \\
\hline 1 & 5 & $\begin{array}{l}\left\{d_{i}, c_{j}, r\right\} \\
\left\{d_{i}, c_{j}, r\right\}\end{array}$ & $\begin{array}{l}\left\{a_{i}, x_{i}, y_{i}\right\} \quad\left\{a_{j}, b_{j}, u_{j}\right\} \text { if } r \in\left\{w_{j}, x_{j}, y_{j}, z_{j}\right\} \\
\left\{a_{i}, x_{i}, y_{i}\right\} \quad\left\{a_{j}, w_{j}, x_{j}\right\} \text { if } r \notin\left\{w_{j}, x_{j}, y_{j}, z_{j}\right\}\end{array}$ \\
\hline 2 & 2 & $\left\{d_{i}, d_{j}, r\right\}$ & $\left\{b_{i}, w_{i}, y_{i}\right\} \quad\left\{b_{j}, w_{j}, y_{j}\right\}$ \\
\hline 2 & 3 & $\left\{d_{i}, d_{j}, r\right\}$ & $\left\{b_{i}, w_{i}, y_{i}\right\}\left\{a_{j}, y_{j}, z_{j}\right\}$ \\
\hline 2 & 4 & $\left\{\begin{array}{l}\left\{d_{i}, d_{j}, r\right\} \\
\left\{d_{i}, d_{j}, r\right\}\end{array}\right.$ & $\begin{array}{ll}\left\{b_{i}, w_{i}, y_{i}\right\} & \left\{a_{j}, w_{j}, x_{j}\right\} \text { if } d_{j} \neq a_{j} \\
\left\{b_{i}, w_{i}, y_{i}\right\} & \left\{b_{j}, w_{j}, y_{j}\right\} \text { if } d_{j}=a_{j}\end{array}$ \\
\hline 2 & 5 & $\left\{\begin{array}{l}\left\{d_{i}, c_{j}, r\right\} \\
\left\{d_{i}, c_{j}, r\right\}\end{array}\right.$ & $\begin{array}{l}\left\{b_{i}, w_{i}, y_{i}\right\} \quad\left\{a_{j}, b_{j}, u_{j}\right\} \text { if } \mathrm{r} \in\left\{w_{j}, x_{j}, y_{j}, z_{j}\right\} \\
\left\{b_{i}, w_{i}, y_{i}\right\} \quad\left\{a_{j}, w_{j}, x_{j}\right\} \text { if } r \notin\left\{w_{j}, x_{j}, y_{j}, z_{j}\right\}\end{array}$ \\
\hline 3 & 3 & $\left\{d_{i}, d_{j}, r\right\}$ & $\left\{a_{i}, y_{i}, z_{i}\right\} \quad\left\{a_{j}, y_{j}, z_{j}\right\}$ \\
\hline 3 & 4 & $\left\{\begin{array}{l}\left\{d_{i}, d_{j}, r\right\} \\
\left\{d_{i}, d_{j}, r\right\}\end{array}\right.$ & $\begin{array}{ll}\left\{a_{i}, y_{i}, z_{i}\right\} & \left\{a_{j}, w_{j}, x_{j}\right\} \text { if } d_{j} \neq a_{j} \\
\left\{a_{i}, y_{i}, z_{i}\right\} & \left\{b_{j}, w_{j}, y_{j}\right\} \text { if } d_{j}=a_{j}\end{array}$ \\
\hline 3 & 5 & $\left\{\begin{array}{l}\left\{d_{i}, c_{j}, r\right\} \\
\left\{d_{i}, c_{j}, r\right\}\end{array}\right.$ & $\begin{array}{l}\left\{a_{i}, y_{i}, z_{i}\right\} \quad\left\{a_{j}, b_{j}, u_{j}\right\} \text { if } r \in\left\{w_{j}, x_{j}, y_{j}, z_{j}\right\} \\
\left\{a_{i}, y_{i}, z_{i}\right\} \quad\left\{a_{j}, w_{j}, x_{j}\right\} \text { if } r \notin\left\{w_{j}, x_{j}, y_{j}, z_{j}\right\}\end{array}$ \\
\hline 4 & 4 & $\left\{\begin{array}{l}\left\{d_{i}, d_{j}, r\right\} \\
\left\{d_{i}, d_{j}, r\right\} \\
\left\{d_{i}, d_{j}, r\right\} \\
\left\{d_{i}, d_{j}, r\right\}\end{array}\right.$ & $\begin{array}{ll}\left\{a_{i}, w_{i}, x_{i}\right\} & \left\{a_{j}, w_{j}, x_{j}\right\} \text { if } d_{i} \neq a_{i}, d_{j} \neq a_{j} \\
\left\{a_{i}, w_{i}, x_{i}\right\} & \left\{b_{j}, w_{j}, y_{j}\right\} \text { if } d_{i} \neq a_{i}, d_{j}=a_{j} \\
\left\{b_{i}, w_{i}, y_{i}\right\} & \left\{a_{j}, w_{j}, x_{j}\right\} \text { if } d_{i}=a_{i}, d_{j} \neq a_{j} \\
\left\{b_{i}, w_{i}, y_{i}\right\} & \left\{b_{j}, w_{j}, y_{j}\right\} \text { if } d_{i}=a_{i}, d_{j}=a_{j}\end{array}$ \\
\hline 4 & 5 & $\left\{\begin{array}{l}\left\{d_{i}, c_{j}, r\right\} \\
\left\{d_{i}, c_{j}, r\right\} \\
\left\{d_{i}, c_{j}, r\right\} \\
\left\{d_{i}, c_{j}, r\right\}\end{array}\right.$ & $\begin{array}{ll}\left\{a_{i}, w_{i}, x_{i}\right\} & \left\{a_{j}, b_{j}, u_{j}\right\} \text { if } d_{i} \neq a_{i}, r \in\left\{w_{j}, x_{j}, y_{j}, z_{j}\right\} \\
\left\{a_{i}, w_{i}, x_{i}\right\} & \left\{a_{j}, w_{j}, x_{j}\right\} \text { if } d_{i} \neq a_{i}, r \notin\left\{w_{j}, x_{j}, y_{j}, z_{j}\right\} \\
\left\{b_{i}, w_{i}, y_{i}\right\} & \left\{a_{j}, b_{j}, u_{j}\right\} \text { if } d_{i}=a_{i}, r \in\left\{w_{j}, x_{j}, y_{j}, z_{j}\right\} \\
\left\{b_{i}, w_{i}, y_{i}\right\} & \left\{a_{j}, w_{j}, x_{j}\right\} \text { if } d_{i}=a_{i}, r \notin\left\{w_{j}, x_{j}, y_{j}, z_{j}\right\}\end{array}$ \\
\hline
\end{tabular}

where $B_{i}$ and $B_{j}$ are both of Type 5 , this being the most complicated case. It is clear that we may assume Type of $B_{i} \leqslant$ Type of $B_{j}$.

We now consider the case where $B_{i}$ and $B_{j}$ are both of Type 5. Suppose first that $r \notin$ $\left\{w_{i}, i, y_{i}, z_{i}, w_{j}, x_{j}, y_{j}, z_{j}\right\}$. Then if $\left\{w_{i}, x_{i}, y_{i}, z_{i}\right\} \cap\left\{w_{j}, x_{j}, y_{j}, z_{j}\right\}=\emptyset$ we may replace $F_{i}$ and $F_{j}$ by $\left\{a_{i}, w_{i}, x_{i}\right\},\left\{a_{j}, w_{j}, x_{j}\right\}$ and $\left\{c_{i}, c_{j}, r\right\}$. Thus, without loss of generality, we may suppose $w_{i}=w_{j}$. If $y_{i} \neq y_{j}$ or $z_{j}$ we may take as our replacements the triples $\left\{b_{i}, w_{i}, y_{i}\right\},\left\{a_{j}, y_{j}, z_{j}\right\}$ and $\left\{c_{i}, c_{j}, r\right\}$. If $y_{i}=y_{j}$ or $z_{j}$, we may take $\left\{a_{i}, y_{i}, z_{i}\right\},\left\{a_{j}, w_{j}, x_{j}\right\}$ 
and $\left\{c_{i}, c_{j}, r\right\}$. There is then no difficulty unless $z_{i}=x_{j}$. If this happens we may take $\left\{a_{i}, w_{i}, x_{i}\right\},\left\{a_{j}, y_{j}, z_{j}\right\}$ and $\left\{c_{i}, c_{j}, r\right\}$ if $x_{i} \neq z_{j}$ and $\left\{b_{i}, w_{i}, y_{i}\right\},\left\{b_{j}, x_{j}, z_{j}\right\}$ and $\left\{c_{i}, c_{j}, r\right\}$ if $x_{i}=z_{j}$. Secondly suppose $r \in\left\{w_{i}, x_{i}, y_{i}, z_{i}, w_{j}, x_{j}, y_{j}, z_{j}\right\}$, say $r \in\left\{w_{i}, x_{i}, y_{i}, z_{i}\right\}$. Then we take as our replacements the triples $\left\{c_{i}, c_{j}, r\right\},\left\{a_{i}, b_{i}, u_{i}\right\}$ and a third triple which is $\left\{a_{j}, b_{j}, u_{j}\right\} \quad$ if $\quad\left\{u_{i}, r\right\} \subset\left\{w_{j}, x_{j}, y_{j}, z_{j}\right\}, \quad\left\{a_{j}, w_{j}, x_{j}\right\} \quad$ if $\quad\left\{u_{i}, r\right\} \cap\left\{w_{j}, x_{j}\right\}=\emptyset$ or $\left\{a_{j}, y_{j}, z_{j}\right\}$ if $\left\{u_{i}, r\right\} \cap\left\{y_{j}, z_{j}\right\}=\varnothing$.

This completes the proof of $(5)$. That equality holds in $(5)$ when $p \equiv 0(\bmod 3)$ is then a consequence of Lemma 1 and the fact that there is a projective plane of order 2. This completes the proof of Theorem 2 .

\section{A related problem}

We discuss briefly a variation of the problem which was raised by our colleague A. Meir. Define a function $H(T, m, p)$ for $T \geqslant m \geqslant 2, p \geqslant 2$ by replacing condition (a) by (a) $^{\prime \prime}|\bigcup \mathscr{F}|=T$, or equivalently, by replacing (a) by the condition that the total number of languages spoken overall is $T$. We have the following theorem.

\section{THEOREM 3.}

$$
H(T, 2, p) \leqslant \min _{1 \leqslant u \leqslant T} \max \{p u, p T /(u+1)\}
$$

Proof. Let $\mathscr{F}$ be a maximal family satisfying (a)", (b)' and (c)'. If there exists $F \in \mathscr{F}$ such that $|F| \leqslant u$, then $F$ covers $\mathscr{F}$ once (since $m=2$ ) and hence $|\mathscr{F}| \leqslant p u$. On the other hand, if $|F| \geqslant u+1$ for all $F \in \mathscr{F}$, then $\mathscr{F}$ will be covered $u+1$ times by $\bigcup \mathscr{F}$ and thus, by Lemma $2,|\mathscr{F}| \leqslant p T /(u+1)$. Thus Theorem 3 is proved.

Note that if we take $u=[\sqrt{ } T]$ in Theorem 3 we get the following upper bound

$$
H(T, 2, p) \leqslant p \sqrt{ } T \text {. }
$$

Equality holds in Theorem 3 for various valves of $T$. For example, if we take $T=t^{2}-t+1$ we get, on choosing $u=t-1$,

$$
H\left(t^{2}-t+1\right) \leqslant\left(t^{2}-t+1\right)(p / t) .
$$

On the other hand, if there exists a projective plane of order $t-1$ and if $p \equiv 0(\bmod t)$, the construction of Lemma 1 may be used to show that equality holds in (9). Furthermore, the argument used to prove Theorem 1 may be used to show that if $T$ and $p$ are sufficiently large, the bound given by (8) is asymptotically correct.

For small values of $T$, we have been able to obtain the following results (in addition to those covered by (9) and the subsequent comment): 


$$
\begin{array}{cc}
H(4,2, p)=5 p / 3 & \text { if } p \equiv 0(\bmod 3) \\
H(5,2, p)=7 p / 4 & \text { if } p \equiv 0(\bmod 4) \\
H(6,2, p)=2 p & \text { if } p \equiv 0(\bmod 2)
\end{array}
$$

We omit the details of the proofs.

We do not know how $H(T, m, p)$ behaves for $m>2$. We have a construction which establishes for each fixed $m$ a lower bound which is approximately $p \sqrt{ }[T(m-1)]$ if $T$ and $p$ are large. It would be natural to conjecture that $(8)$ may be generalized to give $H(T, m, p) \leqslant p \sqrt{ }[T(m-1)]$, but we have not made any progress in this direction.

We conclude by remarking that the problems considered here are closely related to the class of problems discussed in Bollobás (1977) and the references given there.

\section{References}

H. L. Abbott, D. Hanson and A. C. Liu, 'An extremal problem in graph theory', Quart. J. Math. Oxford Ser. (to appear).

Béla Bollobás (1977), 'Disjoint triples in a 3-graph with given maximal degree', Quart. J. Math. Oxford Ser. 28, 81-85.

Mathematics Department Mathematics Department Mathematics Department University of Alberta Technion University of Regina

Edmonton, Canada Haifa

Regina, Canada

T6G 2G1

Israel

S4S 0A2 